\title{
Cyclospora cayetanensis: An Infestation among Diarrheal Children in Kathmandu Valley, Nepal
}

\author{
Balkrishna Bhattachan ${ }^{1,2 *}$, Ganesh Rai ${ }^{1}$, Nabin Narayan Munankarmi ${ }^{3}$,
} Jeevan Bahadur Sherchand ${ }^{2}$

1Shi-Gan International College of Science and Technology (SICOST), Kathmandu, Nepal 2Department of Microbiology of Tribhuvan University Teaching Hospital, Kathmandu, Nepal ${ }^{3}$ Biotechnology Society Nepal (BSN) Kausaltar-3, Bhaktapur, Nepal

\begin{abstract}
Cyclospora cayetanensis, a coccidian parasites which is responsible for recurring diarrhea and gastroenteritis especially among children living under poor hygienic condition in developing country like Nepal. Aimed of this research is to find out the prevalence of intestinal parasites and C. cayetanensis among diarrheal children in a Pediatric Hospital in Kathmandu valley, Nepal. Cross-sectional type of study was done. Altogether 196 stool specimens were collected from June to September 2013 among outpatient diarrheal children in Kanti Children's Hospital. Modified Ziehl Neelson staining method was applied for detection of oocysts of Cyclospora after formal-ether sedimentation. Parasites were detected in 13.7\% (27/196) of stool samples from $\leq 15$ year old diarrheal patients. C. cayetanensis was detected in $4.8 \%$ $(8 / 196)$. In genderwise, infection rate of C. cayetanensis $4.5 \%(5 / 112)$, in male were higher than $3.6 \%(3 / 84)$ in female. In agewise, infection rate of C. cayetanensis in $5.7 \%(3 / 52) 11-15$ year old were highest followed by $4.1 \%(3 / 78)$ in $0-5$ year old and $3.0 \%(2 / 66)$ in $6-10$ year old. In seasonwise, infection rate of Cyclospora was highest in the month of August $7.4 \%$ $(4 / 54)$ followed by $3.9 \%(2 / 51)$ in July, $2.3 \%(1 / 44)$ September and $2.1 \%(1 / 47)$ in June. Altogether five different type of parasites were detected. Infection rate of Giardia lamblia were highest 5.1 \% (10/196) whereas lowest was Cryptosporidium parvum 1.0 \% (2/196). Prevalence of C. cayetanensis is highly probable to infant, neonate, toddler and diarrheal children. Therefore, attention should be made in laboratory investigation of $C$. cayetanensis while suspecting the diarrheal patients infected with other parasites.

Keywords: Cyclospora, Parasites, Modified Ziehl Neelson, Children

*Corresponding author

Email: balkrishna_bhattachan@hotmail.com
\end{abstract}

\section{Introduction}

Cyclospora cayetanensis, is a coccidian parasite which is responsible for prolonged diarrhea in both immune-competent and immunecompromised patients. The pathogenesis and virulence factors phenomenon of $C$. cayetanensis are yet to be defined, but Inflammation and jejunitis had been found out [1]. C. cayetanensis infect human and cause of acute and chronic gastroenteritis [2]. It has been reported from various parts of the world in Southeast Asia [3,4]. The important vehicle for infection is source of water either by ingestion of parasite through contaminated water directly or through contaminated vegetable. Moreover, it has been implicated in outbreaks developing countries (e.g. like Nepal) [5]. In the study of Sherchand and Cross, between 2001-2004 in Nepal showed that contaminated drinking water, raw green vegetables, infected animals were the possible sources of infection with Cyclospora [6].

The Patients suffering from cyclosporisis show the symptoms of an abdominal cramps, diarrhea, fatigue, nausea, and vomiting, chronic watery, anorexia, and weight loss. However, the cause of disease is still unrevealed. This study was carried out at Kanti Children's Hosptial (KCH), Kathmandu Valley, aiming to find out the prevalence of parasites and C. cayetanensis especially diarrheal children.

\section{Material and Methods}

Study site: Cross-sectional type of study was undertaken at $\mathrm{KCH}$ in Kathmandu Valley from June to September 2013.

Collection of data and samples: Demographic data were collected by using a standard questionnaire and with proper instruction $\leq 15$ 
Table 1. Distribution of age and intestinal parasites among diarrheal children from outpatients in Kanti Children's Hospital

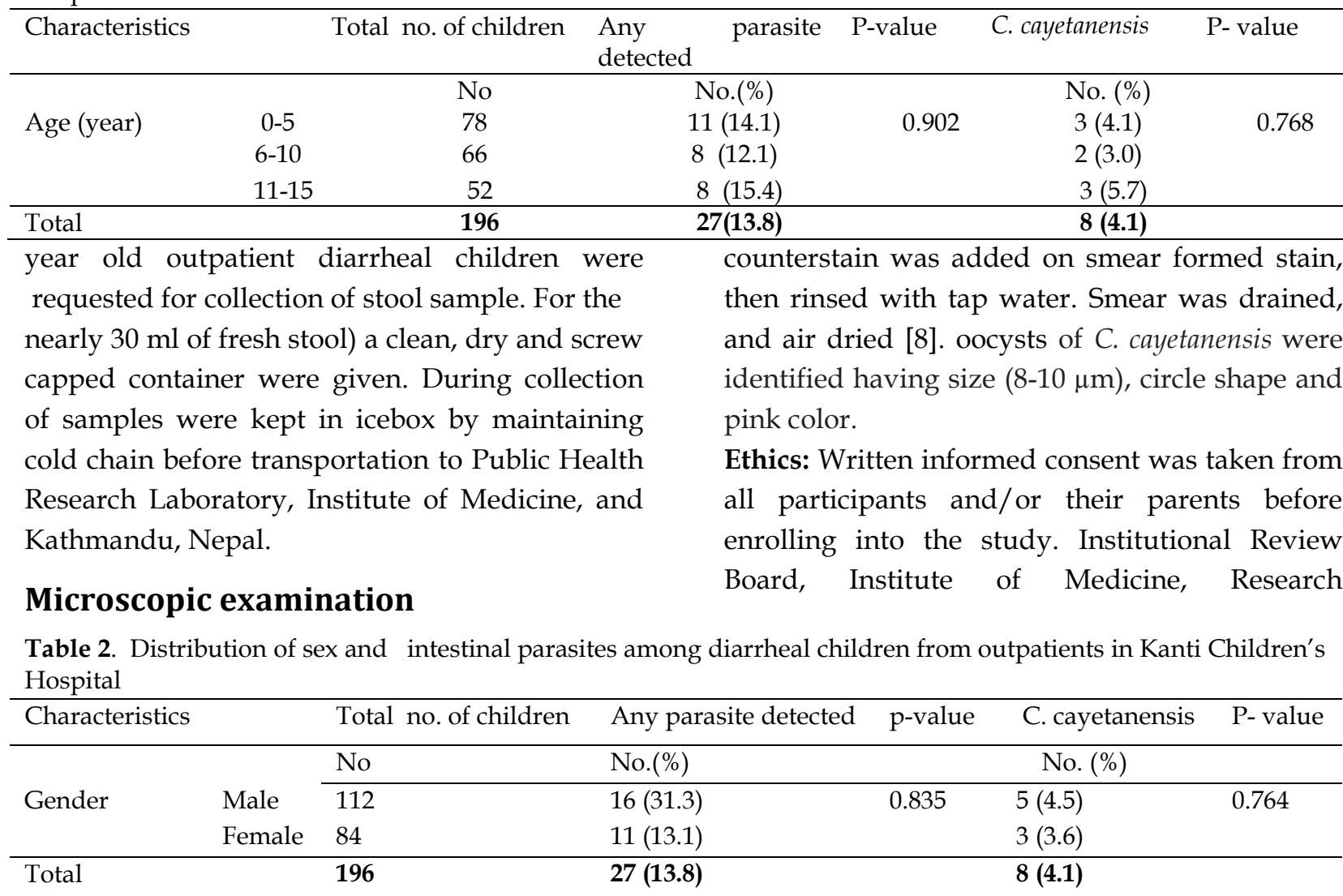

Formal-ether sedimentation Technique: Three $\mathrm{ml}$ of stool samples was taken in test-tube and shaken well then filtered through cotton gauge. After that, $3 \mathrm{ml}$ of ether was added and shaken well then centrifuged. The sediment portion was examined by microscopy after addition of saline solution. Microscopic detection of parasites cyst were carried out under $10 \mathrm{X}$ followed by $40 \mathrm{X}$ objectives [7].

Modified acid fast staining method: The smears were flooded with carbol fuchsin and heated to steaming. It was shaken with tap water, decolorized with 5\% aqueous sulfuric acid, and shake again. Flooded with methylene blue

Table 4: Number of positive and percentage rate of intestinal parasites among diarrheal children were shown in Table 4.

\begin{tabular}{lc}
\hline Name of parasites & Number (\%) \\
\hline Giardia lamblia & $10(5.1)$ \\
Cyclospora cayetanensis & $8(4.8)$ \\
Entamoeba histolytica & $4(2.5)$ \\
Entamoeba coli & $3(1.5)$ \\
Cryptosporidium parvum & $2(1.0)$ \\
\hline
\end{tabular}

*None of multiparasites was detected.
Department, Kathmandu, Nepal was approved this research. Win-pepi statistical software was used for data calculation where $\mathrm{p}$ value of data $<0.05$ considered as statistically significant.

\section{Results}

Parasites were detected in $13.7 \%$ (27/196) of stool samples from $\leq 15$ year diarrheal patients. $C$. cayetanensis was detected in $4.8 \%$ (8/196). Infection rate of C. cayetanensis in $5.7 \%(3 / 52) 11$ 15 years old were highest followed by $4.1 \%$ $(3 / 78)$ in $0-5$ years old and $3.0 \%(2 / 66)$ in $6-10$ years old [Table 1].

In gender, ratio between male and female were 1.3:1, infection rate of C. cayetanensis $4.5 \%$ $(5 / 112)$ in male were higher than $C$. cayetanensis $3.6 \%(3 / 84)$ in female [Table 2].

Table 3: Monthwise- distribution of Cyclospora cayetanensis and total parasites in proportion in stool samples

\begin{tabular}{lcc}
\hline & $\begin{array}{l}\text { Cyclospora } \\
\text { cayetanensis (\%) }\end{array}$ & $\begin{array}{l}\text { Total } \\
\text { parasites (\%) }\end{array}$ \\
\hline Month & & \\
June & 2.1 & 12.9 \\
July & 3.9 & 9.8 \\
August & 7.4 & 18.8 \\
September & 2.3 & 15 \\
\hline
\end{tabular}


In seasonwiser, infection rate of $C$. cayetanensis was highest in the month of August 7.4\% (4/54) followed by $3.9 \%(2 / 51)$ in July, $2.3 \%(1 / 44)$ September and 2.1\% (1/47) in June [Figure 1]. Altogether 6 different types of parasites were detected. Infection rate of Giardia lamblia were highest $5.1 \%(10 / 196)$ whereas lowest was Cryptosporidium paroum $1.0 \%$ (2/196) [Figure 2].

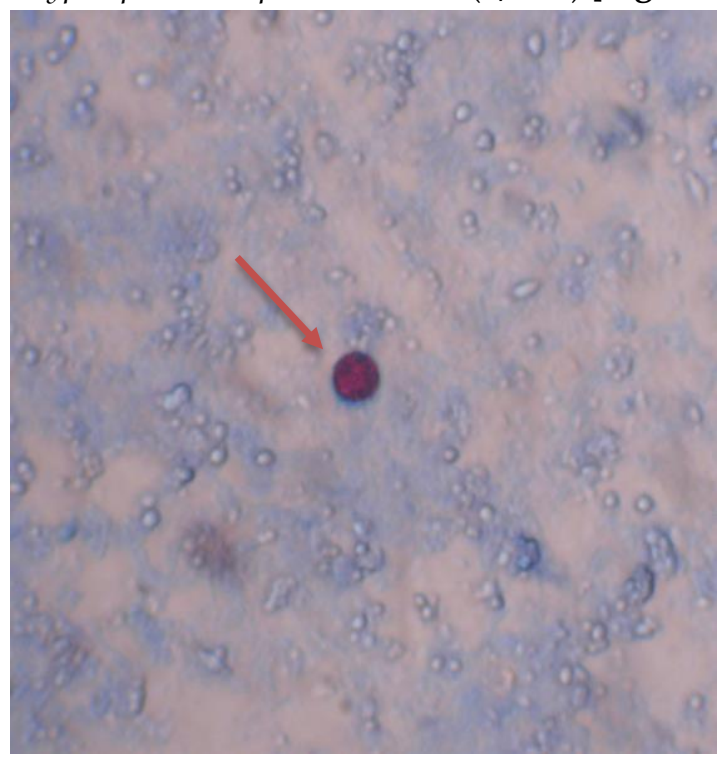

Photograph 1: Oocyst of Cyclospora cayetanensis (ZiehlNeelsen, 100X)

\section{Discussion}

Prevalence of $C$. cayetanensis infections among $\leq 15$ year outpatient diarrheal children in $\mathrm{KCH}$, Kathmandu, Nepal was conducted in this study. Intestinal parasitosis is detected to be highly prevalent in Nepal, small developing country located in south Asia [9]. Infection rate of $C$. cayetanensis and other parasites was higher between 11- 15 years old in diarrheal children. Altogether five species of parasites were detected in $13.7 \%$ of stool samples from $\leq 15$ year old diarrheal patients. Among them, most of prevalence in G. lamblia was detected. In Nepal, C. cayetanensis was detected in $4.8 \%$ of patients. In previous study, $7.9 \%$ were found to be positive for $C$. cayetanensis in diarrheal Children in Nepal [10].

In this study, we could not detect significant differences in prevalence of $C$. cayetanensis among $<15$ year age groups and in gender. In gender, prevalence of C. cayetanensis $4.5 \%$ in male was higher than in female $3.6 \%$. In previous study, prevalence of $C$. cayetanensis in female $(53.4 \%)$ was higher than male (46.6\%) in Nepal [10]. Total of the 60 patients infected with Cyclospora identified in this study, $63.4 \%$ were male and $36.6 \%$ were female in Mexico [11].

In age, prevalence of $C$. cayetanensis in $5.7 \% 11$ 15 years were highest. In previous study, the most of the infection rate of C. cayetanensis infection $(50.7 \%)$ was found between 2-5 year old of age group whereas the lowest prevalence $(4.1 \%)$ were above 28 years of age in Nepal [10]. Cyclospora was most frequently identified in boys of school age (36.7\%) in Mexico [11]. The highest attack rates occur among children older than 18 months where as in our study, however, all age groups may acquire the disease, the highest attack rates was detected among children age between 2 to 5 years $[12,13]$. There is no apparent immunity to infection, and reinfection can occur at all ages $[14,15]$.

Prevalence of $C$. cayetanensis was most in the month of August. The higher distribution of Cyclospora infection in Nepal occurs during summer and rainy season [10]. Their infections were relevantly identified in the rainy season (June-August) in children of Mexico [11]. Cyclosporiasis occurs with high incidence during the rainy seasons from April to June in Peru and May to September in Nepal [1, 2].

In Kanti Children's Hospital, C. cayetanensis infection with diarrheal illness patients is not routinely diagnosed. Thus, adequate diagnosis and treatment are not always conducted promptly. In addition, Cyclospora makes it difficult to include its differential diagnosis due to lack of epidemiologic information. Therefore, our study suggests that there is need of highly specific assays to diagnose Cyclospora infections.

\section{Conclusions}

Prevalence of $C$. cayetanensis is highly probable in infant, neonate, toddler, and children and is one of the important etiologic agents of diarrheal illness among children. It is also equally probable infection in male and female as well as in rainy season. Therefore, attention should be made for laboratory investigation of $C$. cayetanensis while suspecting the diarrheal patients infected with other parasites. 


\section{Author Contributions}

$\mathrm{BB}$ and GR design the proposal and format of research. BB collects the stool sample from hospital and proceeding in Lab of TUTH. JBS and BB and NNM write the article. All authors revised and finalized the draft.

\section{Acknowledgements}

The authors would express sincere thanks to Mrs. Sarmila Tandukar Mr. Kalyan Subedi, Ms. Anisha Shrestha. We are indebted to hospital's staffs and guardian of children who support continually.

\section{Conflict of interest:}

None declared

\section{References}

1. Ortega YR, Roxas CR, Gilman RH, Miller NJ, Cabrera L, Taquiri C, Sterling CR: Isolation of Cryptosporidium paroum and Cyclospora cayetanensis from vegetables collected in markets of an endemic region in Peru. American Journal of Tropical Medicine and Hygiene. 1997 57:683-686.

2. Ortega YR., Sterling CR, Gilman RH, Cama VA, Diaz F.: Cyclospora species-a new protozoan pathogen of humans. N Engl J Med. 1993 328:1308-1312.

3. Soave R: Cyclospora: an overview. Clin Infect Dis. 1996,23: 329-378.

4. Hart AS, Ridinger MT, Soundarajan R, Peters CS, Swiatlo AL, Kocka FE: Novel organism associated with diarrhoea in AIDS. Lancet. 1990 335: 69.

5. Rabold JG, Hoge CW, Shlim DR: Cyclospora outbreak associated with chlorinated drinking water (letter). Lancet. 1994 344: 1360- 1361.

6. Sherchand JB, Cross JH, Jimba M, Sherchand S, Shrestha MP: Study of Cyclospora cayetanensis in health care facilities, sewage water and green leafy vegetables in Nepal. Southeast Asian Journal Trop Med Public Health. 1999; 30: 58-63

7. Bhattachan B, Panta YB, Tiwari S, et al: Intestinal Parasitic Infection Among School children In Chitwan District Of Nepal. J Inst Med. 2015 37(2): 79-84.

8. Garcia LS, Bruckner DA, Brewer TC, et al: Techniques for the recovery and identification of Cryptosporidium oocysts from stool specimens. J Clin Microbiol. 1983; 18:185-190.

9. Rai SK, Hirai K, Abe A, et al: Intestinal parasitosis among school children in a rural hilly area of Dhading District, Nepal. Nepal Med Coll J. 2002 4: 54-58

10. Sherchand JB, Sherchand JB, Cross JH. An epidemiological study of Cyclospora cayetanensis in Nepalese people. J Inst Med.
200729 (1) :8-13.

11. Orozco-Mosqueda GE, Martı́nez-Loya OA, and Ortega YR: Cyclospora cayetanensis in a Pediatric Hospital in Morelia, Mexico. Am Trop Med Hyg. 2014 91(3): 537-540, doi:10.4269/ajtmh.13-0535.

12. Hoge CW, Shlim DR, Ghimire M, Rabold JG, Pandey P, Walch A Rajah JG, Guadio P \& Echeverria P: Placebo controlled trial of cotrimoxazole for Cyclospora infection among travelers and foreign residents in Nepal. Lancet. 1995, 345: 691-693.

13. Schubach TM, Neves ES, Leite AC, Araujo AQC \& de Mouta H: Cyclospora cayetaensis in an asymptomatic patient infected with HIV and HTLV- 1. Transaction of the Royal Society of Tropical Medicine. 1997, 91: 175.

14. Connor BA \& Shlim DR: Food borne transmission of Cyclospora. Lancet. 1995 346: 1634.

15. Caramello P, Brancale T, Forno B, Lucchini A, Macor A, Mazzucco G, Tettoni C \& Ullio A: Clinical and diagnostic aspects of travelers diarrhea due to Cyclospora organisms. Journal of Travel Medicine. 1995 2: 232-234. 